\title{
Development of a mathematical model of dynamic characteristics of a drive with a planetary mechanism
}

\author{
Erkin Nematov ${ }^{1}$, Mukhiddin Khudjaev ${ }^{1, *}$, and Botir Khasanov ${ }^{2}$ \\ ${ }^{1}$ Tashkent State Technical University named after Islam Karimov, 2, Universitetskaya Str., 100174, \\ Tashkent, Uzbekistan \\ ${ }^{2}$ Olmalik branch of Tashkent State Technical University named after Islam Karimov, M. Ulugbek \\ Str., 110100, Olmalik, Uzbekistan
}

\begin{abstract}
The article is devoted to the development of a mathematical model of dynamic characteristics of a drive with a planetary gear mechanism. The subject of research is a drive with a planetary gear mechanism. The following issues were considered in the article: the synthesis of a planetary gear mechanism; the development of a mathematical model that describes the dynamic characteristics of the system; the determination of the dynamic characteristics of a drive with a planetary gear mechanism. Research is based on the method of determining the number of gear teeth; the method of determining the kinetic energy of the James gearbox; the method of determining the dynamic characteristics of an electric motor. The possible number of satellites is given in the article; the pitch radii of the wheels for a given modulus are defined; the moment of inertia of the mechanism reduced to the movable central wheel is determined; a mathematical model of the motion of a drive with a planetary gear mechanism is developed. Equations of motion of a drive with a planetary gear mechanism were obtained. Assuming, in a particular case, all the links of the drive with the planetary gear mechanism as rigid links, a mathematical model was developed for this system, considering the dynamic characteristics of an electric motor. A mathematical model was developed that describes the dynamic characteristics of the system. Analytical solutions for the developed mathematical model are given.
\end{abstract}

\section{Introduction}

Drives with planetary gear mechanisms are widely used in various branches of technology and industry. Simulation of dynamic characteristics and determination of the actual laws of motion of the working bodies play an important role in the design of such drives.

Numerous publications [1-8] are devoted to the study and analysis of various mechanisms of various functions. Analyzing numerous studies aimed at improving the

\footnotetext{
*Correspondind author: mukhiddinkhudjaev@gmail.com
} 
operation of the mechanisms and their wide application, the theoretical foundations and designs of planetary and biplanetary drives of the working bodies of doughing machines were developed in [2]. Mathematical models for controlling the parameters and constraints of the friction mechanism were developed in [3]. The theory and designs of friction mechanisms with controlled friction were developed in [4, 5]. In [6], the analytical implementation of the mathematical model of the controlled motion of the positioning mechanism was developed. A new coaxial eccentric indexing cam mechanism for highspeed automatic units was proposed [7]. A review of the development and state of the kinematics and dynamics of the cam mechanism was conducted [8].

Below, the equations of motion of the grinder drive with a planetary gear mechanism are proposed. Considering, in a particular case, that all the links of the drive with the planetary gear mechanism are rigid, a mathematical model was developed for this system, taking into account the dynamic characteristics of an electric motor. Analytical solutions for the developed mathematical model are given. The obtained research results were used in the design of a universal planetary mill.

\section{Methods}

Research is based on the method of determining the number of gear teeth; the method of determining the kinetic energy of the James gearbox; the method of determining the dynamic characteristics of an electric motor.

\section{Materials}

Determination of the actual laws of motion of the working body of a grinder is a necessary factor in its design. The working body of the grinder receives motion from an asynchronous electric motor through a planetary gearbox. To reduce the number of revolutions of the working disc of the grinder, it was proposed to install a James planetary gearbox. The kinematic diagram of the drive is shown in Fig. 1, where, 1 is a fixed central wheel, 2 is a satellite, 3 is a movable central wheel, 4 is a working body, $\mathrm{H}$ is a carrier.

Consider the methods for determining the number of teeth of a given gearbox [1].

Suppose that it is necessary to design a planetary gear that reproduces the gear ratio $u_{1 H}^{(3)}=a$, where $u_{1 H}^{(3)}$-is the gear ratio between wheels 1 and 3 when the carrier $\mathrm{H}$ is stopped, and equal to $u_{13}^{H}=1-u_{1 H}^{(3)}=b$. In this case $u_{13}^{H}=-\frac{z_{3}}{z_{1}}=b$, then the number of teeth of wheel 3 is

$$
z_{2}=\frac{z_{3}-z_{1}}{2}
$$

or

$$
z_{2}=\frac{b \cdot z_{1}-z_{1}}{2}=\frac{z_{1}(b-1)}{2}
$$

Considering this, we obtain

$$
\frac{z_{3}}{z_{2}}=\frac{2 \cdot b}{(b-1)}
$$


The number of teeth $z_{3}$ should be chosen so that there is no undercut or interference between the teeth. As is known, for normal gear wheels with an engagement angle $\alpha=20^{\circ}$ and a head height coefficient equal to one for external engagement, it is

$$
z_{2}=\frac{z_{1}^{2}-34}{34-2 \cdot z_{1}}
$$

and for internal gearing it is

$$
z_{3} \geq \frac{z_{2}^{2}-34}{2 \cdot z_{2}-34}
$$

Formulas (4), (5) serve to select the permissible number of teeth in the absence of undercutting or jamming of the gear. Calculations according to formula (5) show that the least number of teeth of the little wheel is $z_{2}=17$, while the greater wheel 3 must have the number of teeth $z_{2}=\infty$, that is, wheel 3 turns into a toothed rack.

It should be noted that calculations according to formula (5) can be made up to the number of teeth $z_{2}=26$ since at the number of teeth $z_{2}>26$ for internal gearing, the phenomenon of teeth interference becomes dangerous, and the tooth head of the little wheel is pressed into the tooth head of the large wheel outside the engagement area.

Now let us determine the possible number of satellites $K$ using the conditions of neighborhood and assembly

$$
K<\frac{\pi}{a \sin \frac{z_{2}+2}{z_{1}+z_{2}}}, K=\frac{z_{1}+z_{3}}{n}
$$

$K$ and $n$ in formulas (6) must be integers. Next, we determine the pitch radii of the wheels for a given modulus $m$

$$
z_{1}=\frac{m \cdot z_{1}}{2}, z_{2}=\frac{m \cdot z_{2}}{2}, z_{3}=\frac{m \cdot z_{3}}{2}
$$

For values of $u_{1 H}^{(3)}$ equal to $4,5,6,7,8$, the number of gears teeth $1,2,3$, the number of possible satellites, and the pitch radii of these gears were determined. The results are shown in table 1.

Table 1. Values of geometrical parameters of the James gearbox.

\begin{tabular}{|c|c|c|c|c|c|c|c|c|c|c|}
\hline № & $u_{1 H}^{3}$ & $u_{H 1}^{3}$ & $m$ & $z_{1}$ & $z_{2}$ & $z_{3}$ & $K$ & $R_{1}$ & $R_{2}$ & $R_{3}$ \\
\hline 1 & 4 & 0.25 & 5 & 20 & 20 & 60 & 5 & 50 & 50 & 150 \\
\hline 2 & 5 & 0.2 & 5 & 18 & 25 & 68 & 2 & 10 & 62.5 & 170 \\
\hline 3 & 6 & 0.17 & 5 & 17 & 34 & 85 & 3 & 42.5 & 85 & 212.5 \\
\hline 4 & 7 & 0.143 & 5 & 18 & 45 & 108 & 3 & 45 & 112.5 & 270 \\
\hline 5 & 8 & 0.125 & 5 & 18 & 50 & 120 & 3 & 45 & 125 & 300 \\
\hline
\end{tabular}

We define the kinetic energy of the James gearbox as: 


$$
T=T_{g}+T_{3}+T_{c}+T_{H}
$$

where $T_{g}$-is the kinetic energy of the electric motor rotor; $T_{3}-$ is the kinetic energy of the gear 3; $T_{c}-$ is the kinetic energy of satellites; $T_{H}-$ is the kinetic energy of the carrier. щr

$$
T=\frac{1}{2}\left(J_{g} \cdot \omega^{2}+J_{3} \cdot \omega_{3}^{2}+k \cdot J_{2} \cdot \omega_{2}^{2}+K \cdot m_{2} \cdot R_{H}^{2} \cdot \omega_{H}^{2}+J_{H} \cdot \omega_{H}^{2}\right)
$$

where $J_{g}$ - is the moment of inertia of the electric motor rotor; $J_{3}-$ is the moment of inertia of gear 3 relative to the axis of rotation; $J_{2}-$ is the moment of inertia of the satellite relative to its axis of rotation; $K-$ is the satellite number, $R_{H}$ - is the radius of the satellite center trajectory; $m_{2}-$ is the mass of the satellite, $J_{H}-$ is the moment of inertia of the carrier relative to the axis of rotation.

Let us determine the moment of inertia of the mechanism rated to wheel 3. In this case, the working disk is connected to the carrier, and wheel 3 is the driving link, then

$$
J_{n p 1}=J_{g}+J_{3}+K \cdot J_{2}\left(\frac{\omega_{2}}{\omega_{3}}\right)^{2}+K \cdot m_{2} \cdot R_{H}^{2} \cdot\left(\frac{\omega_{H}}{\omega_{3}}\right)^{2}+J_{H}\left(\frac{\omega_{H}}{\omega_{3}}\right)^{2}+J_{P}\left(\frac{\omega_{H}}{\omega_{3}}\right)^{2}
$$

If we assume that all grinder links are rigid, then the dynamic model of the system is reduced to a single-mass model. The mathematical model for this system, considering the dynamic characteristics of the electric motor, has a well-known form [11]:

$$
\begin{aligned}
& \omega_{\partial}=\omega_{0}\left[1-v_{\partial}\left(M_{\partial}+T_{\partial} \cdot \frac{d M_{\partial}}{d t}\right)\right] \\
& \frac{d \omega_{\partial}}{d t}=\frac{1}{I_{\Pi}}\left(M_{\partial}-M_{c}\right)
\end{aligned}
$$

where $\omega_{\partial}-$ is the angular speed of the electric motor rotor and the disk on which the rotating working bodies are installed; $I_{\Pi}-$ is the total moment of inertia of the electric motor rotor and the disk with the working bodies; $M_{\partial}-$ is the moment developed by the asynchronous electric motor; $M_{c}-$ is the resulting moment from the resistance forces acting on the working body; $\omega_{\partial}$ is the angular speed of the rotor of the electric motor and the disk with working bodies; $\omega_{0}-$ is the angular speed of the ideal no-load speed of the electric motor; $v_{\partial}-$ is the steepness of the static characteristic; $M_{\partial}-$ is the moment developed by the electric motor; $T_{\partial}-$ is the electromagnetic time constant of the engine, depending on the parameters of its electrical circuit.

For an asynchronous electric motor with a squirrel-cage rotor, $v_{\partial}$ and $T_{\partial}$ can be determined by the following dependencies: 


$$
T_{\partial}=\frac{1}{2 \pi f_{c} S_{\kappa}} ; \quad v_{\partial}=\frac{S_{\kappa}}{2 M_{\kappa} \cdot \lambda} ; \quad S_{\kappa}=\left(1-\frac{\omega_{H}}{\omega_{\partial}}\right)\left(\lambda+\sqrt{\lambda^{2}-1}\right)
$$

where $M_{H}$ - is the nominal moment of the motor; $\lambda-$ is the ratio of the critical moment to the nominal moment (overload factor); $f_{c}-$ is the circuit frequency; $S_{\kappa}-$ is the critical slip; $\omega_{H}-$ is the motor rated angular speed.

Taking into account the above, the equation of motion of the grinder drive can be written in the following form:

$$
v_{\partial} T_{\partial} I_{\Pi} \ddot{\omega}_{\partial}+v_{\partial} I_{\Pi} \dot{\omega}_{\partial}+\frac{\omega_{\partial}}{\omega_{0}}=1-v_{\partial}\left(M_{c}+T_{\partial} \cdot \dot{M}_{c}\right)
$$

Let us represent the moment of resistance $M_{c}$ as the sum of the average value of $M_{c}^{*}$ and the variable component $\tilde{M}_{c}(t)$. In a similar way, we represent the angular velocity $\omega_{\partial}$ as $\omega_{\partial}(t)=\omega_{\partial}^{*}+\widetilde{\omega}_{\partial}(t)$. It should be noted that the DC component can be determined from the static characteristic of the electric motor. For this, we assume that $T_{\partial}=0$, then

$$
\omega_{\partial}^{*}=\omega_{0}\left(1-v_{\partial} \cdot M_{c}^{*}\right)
$$

Taking into account expression (9), equation (8) can be written in the following form:

$$
\omega_{\partial}^{*}+2 n \dot{\widetilde{\omega}}_{\partial}+\kappa^{2} \widetilde{\omega}_{\partial}=W(t)
$$

where

$$
n=\frac{1}{2 T_{\partial}} ; \kappa^{2}=\frac{1}{v_{\partial} \cdot T_{\partial} \cdot I_{\Pi} \cdot \omega_{0}} ; W(t)=-\frac{1}{I_{\Pi}}\left(\frac{M_{c}}{\widetilde{T}_{\partial}}+\dot{\tilde{M}}_{c}\right)
$$

To solve equation (6), we use the Fourier series [2, 3]. As is well known, if the force $Q(t)$ is a periodic function of the time period $\tau=2 \pi / \omega$, then under general assumptions, always satisfied in real dynamical systems, it can be expanded in a Fourier series:

$$
Q(t)=Q_{0}+\sum_{j=1}^{\infty} Q_{j} \cdot \sin \left(j \omega t+\theta_{j}\right)
$$

where

$$
\begin{gathered}
Q_{0}=\frac{1}{\tau} \int_{0}^{\tau} Q(t) d t ; \quad Q_{j C}=\frac{2}{\tau} \int_{0}^{\tau} Q(t) \cos j \omega t \cdot d t ; \quad Q_{j S}=\frac{2}{\tau} \int_{0}^{\tau} Q(t) \sin j \omega t \cdot d t ; \\
Q_{j}=\sqrt{Q_{j C}^{2}+Q_{j S}^{2}} ; \cos \theta_{j}=\frac{Q_{j S}}{Q_{j}} ; \sin \theta_{j}=\frac{Q_{j C}}{Q_{j}} .
\end{gathered}
$$

The individual terms of the series (11) are called harmonics. In linear systems, the principle of superposition is valid, according to which forced oscillations from each 
harmonic can be determined separately, and the results can be summed. Based on this, for the case of a harmonic exciting force, the expression for forced vibrations is written in the following form

$$
q=A_{0}+\sum_{j=1}^{\infty} A_{j} \sin \left(j \omega t+\theta_{j}-\Delta_{j}\right)
$$

where $A_{0}=Q_{0} / c ; A_{j}=A_{j c m} K_{g} ; A_{j c m}=Q_{j} / c$.

The dynamic factor $K_{g}$ and phase shift can be determined using the following formulas:

$$
K_{g}=\frac{1}{\sqrt{\left(1-j^{2} z^{2}\right)^{2}+4 \delta^{2} j^{2} z^{2}}} ; \quad \operatorname{tg} \Delta_{j}=\frac{2 j z \delta}{1-j^{2} z^{2}}
$$

where $\delta=\frac{n}{k}-$ is called the fraction of critical damping, bearing in mind that at $\delta=1$ the dynamic process under free vibrations goes from oscillatory process to periodic $z=\omega / k$. It is known that the use of Fourier series turns out to be more effective with wellconverging functions $Q(t)$ and at a relatively small excess of the frequency of free oscillations " $k$ " over fundamental excitation frequency $\omega=2 \pi / r$.

In the grinder of mineral raw materials, considered in the article, an asynchronous electric motor is used with a power of $N=18,5 \mathrm{~kW}$; rated speed of $n_{H}=3000 \mathrm{rpm}$. Let us determine the angular velocity of the electric motor $\omega_{\partial}$.

$$
\begin{gathered}
J_{i}=0,1 \mathrm{~kg} \cdot \mathrm{m}^{2} ; \\
M_{C}=(50-18,2 \cos \omega \cdot t-15 \sin \omega \cdot t+9 \cos 2 \omega \cdot t-20 \sin 2 \omega \cdot t) N \cdot m \\
\text { at } \omega=90 \mathrm{~s}^{-1} .
\end{gathered}
$$

Determine nominal moment

$$
\grave{I}_{i}=9549 \frac{N}{n_{H}}=60,1 N \cdot m
$$

Nominal slip is

$$
\begin{gathered}
S_{H}=1-\frac{n_{H}}{n_{0}}=0,02 ; S_{k} \approx S_{H}\left(\lambda+\sqrt{\lambda^{2}-1}\right)=0,057 ; \\
v=\frac{S_{k}}{2 M_{H} \cdot \lambda}=2,96 \cdot 10^{-4} \frac{1}{N \cdot m} . \\
T_{g}=\frac{1}{2 \pi \cdot f_{c} \cdot S_{k}}=0,056 \mathrm{~s},
\end{gathered}
$$




$$
\omega_{g}^{*}=\omega_{0}\left(1-v_{g} M_{c}^{*}\right)=309,5 s^{-1},
$$

here $\omega_{0}=\frac{\pi n_{0}}{30}=314,2 \mathrm{~s}^{-1} ; M_{c}^{*}=50 \mathrm{~N} \cdot \mathrm{m} \mathrm{s}^{-3}$,

$$
W=Q_{1} \sin \left(\omega t+\theta_{1}\right)+Q_{2} \sin \left(2 \omega t+\theta_{2}\right),
$$

where $Q_{1}=40000 \mathrm{~s}^{-3} ; Q_{2}=38000 \mathrm{~s}^{-3} ; \theta_{1}=89^{\circ} ; \theta_{2}=32^{\circ}$.

The solution to the differential equation $(10)$ is

$$
\omega_{g}=\omega_{1} \sin \left(\omega t+\theta_{1}-\Delta_{1}\right)+\omega_{2} \sin \left(2 \omega t+\theta_{2}-\Delta_{2}\right)
$$

where $\omega_{1}=\frac{Q_{1}}{\sqrt{\left(k^{2}-\omega^{2}\right)^{2}+4 n^{2} \omega^{2}}} ; \quad \omega_{2}=\frac{Q_{2}}{\sqrt{\left(k^{2}-4 \omega^{2}\right)^{2}+16 n^{2} \omega^{2}}} ;$

$$
\begin{aligned}
& \Delta_{1}=\operatorname{arctg} \frac{2 n \omega}{k^{2}-\omega^{2}} ; \Delta_{2}=\operatorname{arctg} \frac{4 n \omega}{k^{2}-4 \omega^{2}} ; \\
& k=\frac{1}{v_{g} T_{g} J_{n} \omega_{0}}=43,85 s^{-1} ; n=\frac{1}{2 T_{g}}=8,95 \mathrm{~s}^{-1} ;
\end{aligned}
$$

\section{Results and discussion}

On the basis of the analytical expressions obtained, the pattern of changes in the angular velocity of the driving link was determined for the steady-state operation of the grinder. The calculations showed that with the above parameters, the peak-to-peak amplitude of the angular velocity of the electric motor rotor is approximately $18.6 \mathrm{~s}^{-1}$.

The results obtained were used in the design of a universal planetary mill.

Based on the study conducted, the following results (conclusions) were drawn:

1. A mathematical model of the motion of a drive with a planetary gear mechanism was developed.

2. On the basis of the analytical expressions obtained with the developed mathematical model, the pattern of changes in the angular velocity of the driving link in the steady-state operation of the grinder was determined.

3 . The results obtained were used in the design of a universal planetary mill.

\section{References}

1. I.I. Artobolevsky, The theory of mechanisms and machines (Nauka, M., 1988)

2. R.I. Karimov, Theoretical foundations and designs of planetary and biplanetary drives of working bodies of doughing machines: Monograph (Tashkent State Technical University, Tashkent, 2013)

3. K.A. Karimov, R.I. Karimov, A.Kh. Akhmedov, Development of the theoretical foundations of mechanisms with controlled parameters and constraints. Monograph (Tashkent State Technical University, Tashkent, 2016)

4. K.A. Karimov, A.Kh. Umurzakov, Theory and design of friction mechanisms with controlled friction. Monograph (Tashkent State Technical University, Tashkent, 2015) 
5. K.A. Karimov, R.F. Nagaev, H.H. Usmonhodjaev, Proceedings of the Sixth World Congress on the Theory of Machines and Mechanism. New Delhi. Ind. 1, 230-232 (1984)

6. K.A. Karimov, U.A. Abduvaliev, A.X. Ahmedov, European Applied Sciences 3, 61-63 (2015) ISSN 2195 - 2183

7. Yuhu Yang, Mechanism and Machine Theory 132, 1-12 (2019) https://doi.org/10.1016/j.mechmachtheory.2018.10.012

8. Fan Y. Chen, Mechanism and Machine Theory 12(3), 201-224 (1977) https://doi.org/10.1016/0094-114X(77)90020-9 